\title{
NUMERICAL RESULT FOR VARIATIONAL INEQUALITY PROBLEMS WITH EQUALITY CONSTRAINT
}

\author{
Pham Van Loi \\ Electric Power University, 235 Hoang Quoc Viet Rd., \\ Cau Giay Dist., Ha Noi, Vietnam
}

Received 18 December 2007

\begin{abstract}
In this paper we present some numerical results for illustrating a theoretical results obtained in our investigation in the field of variational inequality problems with constraint in the form of operator equation involving monotone operator.
\end{abstract}

Key words: Monotone operators, Fréchet differentiable, weakly lower semi-continuous proper convex functional, and regularization.

22000 Mathematics Subject Classification: 47H17; CR: G 1.8.

\section{INTRODUCTION}

Many problems arising in mathematical physics and mechanics have been formulated in the following abstract form: find an element $x \in S_{0}$ such that

$$
\varphi\left(x_{0}\right)=\min _{x \in S_{0}} \varphi(x)
$$

where $\varphi$ is a weakly lower semicontinuous and properly convex functional on a real re exive Banach space $X$ with the norm denoted by $\|\cdot\|$, and $S_{0}$ is a convex and closed subset of the space $X$. If we denote by $A(x)$ the subdifferential of the functional $\varphi$ at the point $x$, then problem (1.1) is equivalent to the variational inequality

$$
\left\langle A(x), x-x_{0}\right\rangle \geq 0 \quad \forall x \in S_{0}, \quad x_{0} \in S_{0},
$$

where the symbol $\left\langle x^{*}, x\right\rangle$ denote the value of the linear and continuous functional $x^{*} \in X^{*}$ at the point $x \in X$, and $X^{*}$ is the adjoint space of the space $X$. For the sake of simplicity, the norm of $X$ will be denoted by the symbol $\|$.$\| , too. When \varphi$ is a convex functional and $S_{0}$ is the set of solutions of the operator equation

$$
F(x)=f_{0}, \quad f_{0} \in X^{*},
$$


where $F: X \rightarrow X^{*}$ is a monotone and continuous operator, and $f_{0} \in R(F)$, the range of $F$, it is well known (see [1]) that Problem (1.3), without additional conditions on the structure of $F$ such as strong or uniform monotonicity, is ill-posed. By this we mean that solutions of (1.3) do not depend continuously on the data $\left(F, f_{0}\right)$. So, Problem (1.2)-(1.3) in this case is ill-posed, too. In numerical computation, we often know the approximations $\left(F_{h}, f_{\delta}\right)$ of $\left(F, f_{0}\right)$ such that

$$
\left\|F(x)-F_{h}(x)\right\| \leq h g(\|x\|), \quad \forall x \in X, \quad\left\|f_{\delta}-f_{0}\right\|<\delta, \quad h, \delta \rightarrow 0,
$$

where $g(t)$ is some real continuous and positive function. Assume that $A$, the Fréchet derivative of $\varphi$ satisfies the condition

$$
\langle A(x)-A(y), x-y\rangle \geq m_{A}\|x-y\|^{s} \quad \forall x, y \in X, \quad m_{A}>0,
$$

for $s \in R, \quad 2 \leq s<+\infty$.

\section{THEORETICAL RESULTS}

A regularized solution of the stated problem can be constructed by the following operator equation (see[3])

$$
F_{h}(x)+\alpha A(x)=f_{\delta} \quad \alpha>0 .
$$

Assumption 2.1 There is a positive constant $\tilde{r}$ such that

$$
\left\|F(y)-F_{h}(x)-F^{\prime}(x)(y-x)\right\| \leq \tilde{r}\|F(y)-F(x)\|,
$$

for $y$ belonging to some neighbourhood of $S_{0}, x \in S_{0}$.

We have the following results (see [3], [4]).

Theorem 2.1 Assume that the following conditions hold:

(i) $F$ is Fréchet differentiable at some neighbourhood of $S_{0}$ with Assumption 2.1, for $x=x_{0}$;

(ii) there exists an elements $z \in X$ such that

$$
F^{\prime}\left(x_{0}\right)^{*} z=A\left(x_{0}\right) \text {; }
$$

(iii) the parameter $\alpha$ is chosen such that $\alpha \sim(\delta+h)^{\rho}, \quad 0<\rho<1$.

Then,

$$
\begin{gathered}
\left\|x_{\alpha}^{r}-x_{0}\right\|=O\left((\delta+h)^{\beta_{1}}\right), \\
\beta_{1}=\min \left\{\frac{1-\rho}{s-1}, \frac{\mu}{s}\right\} .
\end{gathered}
$$


For computation, consider the finite-dimensional problems

$$
F_{h}^{n}\left(x_{\alpha, n}^{r}\right)+\alpha A^{n}\left(x_{\alpha, n}^{r}\right)=f_{\delta}^{n} ;
$$

with $F_{h}^{n}=P_{n}^{*} F_{h} P_{n}, A^{n}=P_{n}^{*} A P_{n}, f_{h}^{n}=P_{n}^{*} f_{\delta}$, where $P_{n}: X \rightarrow X_{n}$, denotes the linear projection from $X$ onto its subspace $X_{n}$ satisfying the condition

$$
X_{n} \subset X_{n+1}, \quad P_{n} x \rightarrow x, \quad n \rightarrow+\infty, \quad \forall x \in X
$$

and $P_{n}^{*}$ is the adjoint of $P_{n},\left\|P_{n}\right\| \leq c_{0}, c_{0}$ is a positive constant. Without loss of generality, suppose that $\left\|P_{n}\right\|=1$.

Set

$$
\begin{gathered}
\gamma_{n}(x)=\left\|\left(I-P_{n}\right) x\right\|, \quad x \in X, \quad \gamma_{n}^{*}(f)=\left\|\left(I^{*}-P_{n}^{*}\right) f\right\| \\
\gamma_{n}=\max \left\{\gamma_{n}(x), \quad x \in S_{0}, \quad \gamma_{n}^{*}(f)\right\}
\end{gathered}
$$

where $I$ and $I^{*}$ denote the identity operators in $\mathrm{X}$ and $\mathrm{X}^{*}$, respectively. By Theorem 1.1 there exists $x_{\alpha, n}^{r}$ satisfying (1.7) for every $\alpha>0$.

Suppose that

$$
\|A(x)-A(y)\| \leq C(R)\|x-y\|^{v}
$$

where $C(R), R>0$, is a positive increasing function on $R=\max \{\|x\|,\|y\|\} \quad$ such that

$$
\limsup _{t \rightarrow+\infty} \frac{C(t)}{t^{\mu_{0}}} \leq q_{2}<+\infty, \quad 0<\mu+\mu_{0}<s-1,
$$

and $A$ is continuous with $A(0)=0$.

The convergence of the sequence $\left\{x_{\alpha, n}^{r}\right\}$ to $x_{0}$ is established by the following therorem.

Theorem 2.2 Suppose that the following conditions hold:

(i) $F$ is Fréchet differentiable at some neighbourhood of $S_{0}$ with Assumption 2.1, for $x=x_{0}$;

(ii) $F_{h}\left(X_{n}\right)$ are contained in $X_{n}^{*}$ for suficiently large $n$ and small $h$;

(iii) there exists an elements $z \in X$ such that

$$
F^{\prime}\left(x_{0}\right)^{*} z=A\left(x_{0}\right)
$$

(iv) the parameter $\alpha$ is chosen such that $\alpha \sim\left(\delta+h+\gamma_{n}\right)^{\rho}, \quad 0<\rho<1$.

Then, we have 


$$
\left\|x_{\alpha, n}^{r}-x_{0}\right\|=O\left(\left(\delta+h+\gamma_{n}\right)^{\beta_{1}}+\gamma_{n}^{\mu /(s-1)}\right) .
$$

\section{NUMERICAL EXAMPLE}

For illustration, we consider problems (1.2) - (1.3) when

$$
\varphi(x)=\frac{1}{2}\left\|x-x_{*}\right\|_{L_{2}[0 ; 1]}^{2}
$$

and $F$ is the subdifferentional of the functional $\psi(x), \psi(x)=\tilde{\psi}(\langle K x, x\rangle)$, where

$$
\tilde{\psi}(t)=\left\{\begin{array}{lll}
0 & \text { if } & t \leq a_{0} ; \\
\frac{\left(t-a_{0}\right)^{2}}{2 h} & \text { if } & a_{0}<t \leq a_{0}+h ; \\
t-a_{0}-\frac{h}{2} & \text { if } & a_{0}+h<t ;
\end{array}\right.
$$

$h$ is positive parameter, $a_{0}$ is fixed positive number,

$$
K x(t)=\int_{0}^{1} k(t, s) x(s) d s, \quad x(s) \in L_{2}[0 ; 1]
$$

with

$$
k(x, s)=\left\{\begin{array}{lll}
t(1-s) & \text { if } \quad t \geq s \\
s(1-t) & \text { if } \quad t<s
\end{array}\right.
$$

Then, $K$ is the linear, continuous and nonnegative operator on $L_{2}[0 ; 1]$. Since $\langle K x, x\rangle$ is a convex functional and $\psi(t)$ is a convex function then $\psi(\langle K x, x\rangle)$ is a convex functional, too. Therefore $F(x)=\partial \psi(x)$ is monotone operator and

$$
F(x)=\partial \psi(x)=\frac{1}{2} \tilde{\psi}^{\prime}(\langle K x, x\rangle) K x .
$$

From (3.2) and (3.3) it follows

$$
F(x)=\left\{\begin{array}{lll}
0 & \text { if } & \langle K x, x\rangle \leq a_{0} ; \\
\frac{\langle K x, x\rangle}{h} K x & \text { if } & a_{0}<\langle K x, x\rangle \leq a_{0}+h ; \\
K x & \text { if } & a_{0}+h<\langle K x, x\rangle .
\end{array}\right.
$$

If choosing $x_{*}=0$, then solution of problem (1.3) is $x_{*}=0$. 
In numerical computation, we choose method as follows:

+) Divide $[0 ; 1]$ in to $n$ equal parts by divide points

$$
t_{i}=i h ; \quad h=\frac{1}{n} ; \quad i=0,1, . ., n .
$$

$+) \int_{0}^{1} k(t, s) x(s) d s$ replaced by

$$
T_{i}=\sum_{j=0}^{n} b_{j} k\left(t_{i}, t_{j}\right) x\left(t_{j}\right) ; \quad i=0,1, \ldots, n,
$$

where $b_{0}=b_{n}=\frac{h}{2}, b_{1}=b_{2}=\ldots=b_{n-1}=h$.

$+) \int_{0}^{1} \int_{0}^{1} k(t, s) x(s) x(t) d s d t$ replaced by

$$
T=\sum_{i=0}^{n} b_{i}\left(\sum_{j=0}^{n} b_{j} k\left(t_{i}, t_{j}\right) x\left(t_{j}\right)\right) x\left(t_{i}\right) .
$$

Problem (3.1) is appproximated by

$$
F\left(x\left(t_{i}\right)\right)=\left\{\begin{array}{lll}
0 & \text { if } & T \leq a_{0} ; \\
\frac{T}{h} T_{i} & \text { if } & a_{0}<T \leq a_{0}+h ; \\
T_{i} & \text { if } & a_{0}+h<T .
\end{array}\right.
$$

$(i=0,1, \ldots, n)$

Problem (3.4) is an algebraic equations systems ( $n+1$ equations). To solve problem (3.4), we use the iterative regularization method as follows (see [2])

$$
z_{k+1}=z_{k}-\beta_{k}\left(F_{h}^{n}\left(z_{k}\right)+\alpha_{k} z_{k}\right) \quad k=0,1, \ldots
$$

with $\beta_{k}=(1+k)^{-1 / 2} ; \alpha_{k}=(1+k)^{-p}, 0<p<1$, choosing $p=\frac{1}{4}$.

We apply the iterative regularization method according to (2.5) to find approximative solutions as follows:

$\left.1^{0}\right)$ Choosing $x=\left(x_{0}, x_{1}, \ldots, x_{n}\right) \neq(0,0, \ldots, 0), a_{0}, h$.

$2^{0}$ ) Computing $T_{n}$ and $T$.

$\left.3^{0}\right)$ Check conditions according to $T$ :

+) If $T \leq a_{0}, x=0$ and stop iterative steps; 
+) If $T>a_{0}+h, x=x-\beta_{k}\left(K x+\alpha_{k} z_{k}\right)=x-e$, moving to step $4^{0}$;

+) If $a_{0}<T \leq a_{0}+h, x=x-\beta_{k}\left(\frac{T-a_{0}}{h} K x-\alpha_{k} z_{k}\right)=x-e$, moving to step $4^{0}$.

$4^{0}$ ) Check $\|e\| \leq \varepsilon$ ?

+ If it is true, $x$ is approximative to the need, and then stop.

$+)$ If it is false, moving to step $2^{0}$.

Result of computation are showed in table 1 :

Table 1.

\begin{tabular}{ccccccc}
\hline $\mathbf{k}$ & $\mathbf{t}=\mathbf{0 . 0}$ & $\mathbf{t}=\mathbf{0 . 2}$ & $\mathbf{t}=\mathbf{0 . 4}$ & $\mathbf{t}=\mathbf{0 . 6}$ & $\mathbf{t}=\mathbf{0 . 8}$ & $\mathbf{t}=\mathbf{1 . 0}$ \\
\hline 1 & 0.0323223305 & 0.0323164376 & 0.0323134912 & 0.0323134912 & 0.0323164376 & 0.0323223305 \\
2 & 0.0226556990 & 0.0226501456 & 0.0226473689 & 0.0226473689 & 0.0226501456 & 0.0226556990 \\
3 & 0.0167460181 & 0.0167414661 & 0.0167391902 & 0.0167391902 & 0.0167414661 & 0.0167460181 \\
4 & 0.0128547780 & 0.0128511166 & 0.0128492859 & 0.0128492859 & 0.0128511166 & 0.0128547780 \\
5 & 0.0101523938 & 0.0101494314 & 0.0101479502 & 0.0101479502 & 0.0101494314 & 0.0101523938 \\
6 & 0.0081985647 & 0.0081961396 & 0.0081949270 & 0.0081949270 & 0.0081961396 & 0.0081985647 \\
7 & 0.0067406309 & 0.0067386205 & 0.0067376154 & 0.0067376154 & 0.0067386205 & 0.0067406309 \\
8 & 0.0056246518 & 0.0056229656 & 0.0056221226 & 0.0056221226 & 0.0056229656 & 0.0056246518 \\
9 & 0.0047522638 & 0.0047508343 & 0.0047501195 & 0.0047501195 & 0.0047508343 & 0.0047522638 \\
10 & 0.0040581302 & 0.0040569067 & 0.0040562950 & 0.0040562950 & 0.0040569067 & 0.0040581302 \\
11 & 0.0034974310 & 0.0034963749 & 0.0034958468 & 0.0034958468 & 0.0034963749 & 0.0034974310 \\
12 & 0.0030385705 & 0.0030376519 & 0.0030371926 & 0.0030371926 & 0.0030376519 & 0.0030385705 \\
13 & 0.0026587492 & 0.0026579447 & 0.0026575425 & 0.0026575425 & 0.0026579447 & 0.0026587492 \\
14 & 0.0023411783 & 0.0023404695 & 0.0023401151 & 0.0023401151 & 0.0023404695 & 0.0023411783 \\
15 & 0.0020732737 & 0.0020726458 & 0.0020723318 & 0.0020723318 & 0.0020726458 & 0.0020732737 \\
16 & 0.0018454539 & 0.0018448948 & 0.0018446152 & 0.0018446152 & 0.0018448948 & 0.0018454539 \\
17 & 0.0016503209 & 0.0016498208 & 0.0016495707 & 0.0016495707 & 0.0016498208 & 0.0016503209 \\
18 & 0.0014820908 & 0.0014816415 & 0.0014814168 & 0.0014814168 & 0.0014816415 & 0.0014820908 \\
19 & 0.0013361899 & 0.0013357848 & 0.0013355823 & 0.0013355823 & 0.0013357848 & 0.0013361899 \\
20 & 0.0012089650 & 0.0012085984 & 0.0012084151 & 0.0012084151 & 0.0012085984 & 0.0012089650 \\
21 & 0.0010974700 & 0.0010971372 & 0.0010969708 & 0.0010969708 & 0.0010971372 & 0.0010974700 \\
22 & 0.0009993093 & 0.0009990062 & 0.0009988547 & 0.0009988547 & 0.0009990062 & 0.0009993093 \\
\hline & & & & &
\end{tabular}

Remarks. From the numerical table we have the following remarks:

- The number of iterations depends on the the choice of values of $p$.

- If number of iterations is large, approximation solution is near the exact solution of the original problem.

\section{REFERENCES}

1. Ya.I. Alber (1975), On solving nonlinear equations involving monotone operators in Banach 
spaces, Sibirskii Math. J., pp. 26, 3-11 (in Russian).

2. Bakushinsky, A.B. (1976), A regularizing algorithm based on the Newton-kantorovich method for solving variational inequalities, J. of Math. Comp. and Math. Physics, V. 16, N. 6, pp. 1397-1404 (in Russian).

3. Nguyen Buong and Pham Van Loi (2004), On parameter choice and convergence rates for a class of ill-posed variational inequalities, J. of Math. Comp. and Math. Physics, V. 44, pp. 1735-1744 (in Russian).

4. Pham Van Loi and Nguyen Buong (2005), About convergence and finite-dimensional approximation for a class of ill-posed variational inequalities, Advances in Natural Sciences, Vol. 6, No. 4, pp. 321-328. 